\title{
Characterizing virus-induced gene silencing at the cellular level with in situ multimodal imaging
}

\author{
Sadie J. Burkhow ${ }^{1,2}$, Nicole M. Stephens ${ }^{1,2}$, Yu Mei $^{3}$, Maria Emilia Dueñas ${ }^{1,2}$, Daniel J. Freppon 1,2, Geng Ding ${ }^{4}$, \\ Shea C. Smith ${ }^{5}$, Young-Jin Lee ${ }^{1,2}$, Basil J. Nikolau ${ }^{4}$, Steven A. Whitham ${ }^{3}$ and Emily A. Smith ${ }^{1,2^{*}}$ (D)
}

\begin{abstract}
Background: Reverse genetic strategies, such as virus-induced gene silencing, are powerful techniques to study gene function. Currently, there are few tools to study the spatial dependence of the consequences of gene silencing at the cellular level.

Results: We report the use of multimodal Raman and mass spectrometry imaging to study the cellular-level biochemical changes that occur from silencing the phytoene desaturase ( $p d s)$ gene using a Foxtail mosaic virus (FoMV) vector in maize leaves. The multimodal imaging method allows the localized carotenoid distribution to be measured and reveals differences lost in the spatial average when analyzing a carotenoid extraction of the whole leaf. The nature of the Raman and mass spectrometry signals are complementary: silencing $p d s$ reduces the downstream carotenoid Raman signal and increases the phytoene mass spectrometry signal.

Conclusions: Both Raman and mass spectrometry imaging show that the biochemical changes from FoMV-pds silencing occur with a mosaic spatial pattern at the cellular level, and the Raman images show carotenoid expression was reduced at discrete locations but not eliminated. The data indicate the multimodal imaging method has great utility to study the biochemical changes that result from gene silencing at the cellular spatial level of expression in many plant tissues including the stem and leaf. Our demonstrated method is the first to spatially characterize the biochemical changes as a result of VIGS at the cellular level using commonly available instrumentation.
\end{abstract}

Keywords: RNA silencing, Foxtail mosaic virus, Phytoene desaturase, Subcellular Raman imaging, Mass spectrometry imaging, Whole-plant analysis, Biochemical characterization, Mosaic spatial pattern, Carotenoids

\section{Background}

Reverse genetics techniques, such as RNA silencing, have been widely used over the past 20 years to generate loss-of function phenotypes that provide insight into the functions of silenced genes. Virus-induced gene silencing (VIGS) is a method of RNA silencing that takes advantage of the plant's natural antiviral defense mechanisms. VIGS requires a modified viral vector that carries RNA or DNA fragments corresponding to the plant target

\footnotetext{
${ }^{*}$ Correspondence: esmith1@iastate.edu

${ }^{1}$ The Ames Laboratory, U.S. Department of Energy, lowa State University, Ames, IA 50011-3111, USA

Full list of author information is available at the end of the article
}

gene(s). The recombinant virus replicates and moves systemically throughout the plant. Meanwhile the antiviral RNA silencing system is activated against the viral genetic template, which also encompasses the target plant gene fragment, resulting in the silencing of the target gene. The use of VIGS technologies addresses the need for rapid and potentially high-throughput methods for testing gene functions in a wide variety of monocot and dicot plant species [1-11].

Foxtail mosaic virus (FoMV), belonging to the Potexvirus genus, was recently developed as a VIGS vector for use in maize and other important monocot crop species such as wheat $[1,2]$. The genome organization of FoMV and other potexviruses consists of five major 
open reading frames (ORFs) encoding: RNA polymerase (ORF1), the triple gene block (ORF2-4), and the coat protein (ORF5) [10, 12], all of which are essential for virus survival and function. In addition, FoMV encodes a unique $5 \mathrm{~A}$ protein that is not essential for replication or viral infection [12]. Mei et al. [1] developed a DNAbased full-length FoMV VIGS vector by inserting a cloning site after the coat protein. This FoMV vector was used to silence phytoene desaturase ( $p d s)$ and other genes in sweet corn and the B73 inbred line of yellow dent corn. Silencing $p d s$ in tobacco leaves has been shown to produce an easily observed variegated white phenotype [11]. The $p d s$ enzyme along with other desaturases and isomerases convert the colorless phytoene molecule to downstream carotenoids (Fig. 1) [13-18]. These downstream carotenoids have multiple conjugated double bonds that lead to the absorption of light in the visible region ( 390-700 nm). Zhang et al. [19] utilized a Bean pod mottle virus VIGS vector to silence $p d s$ within soybean leaves. They tagged this VIGS vector with green fluorescent protein (GFP), and confirmed via fluorescence that the vector was spatially correlated to the visual mosaic phenotype produced from silencing $p d s$ [19]. Juvale et al. [20] performed a similar experiment with transgenic soybeans that constitutively expressed a GFP transgene in all tissues to measure GFP VIGS from a Bean pod mottle virus vector [20]. They determined that the GFP transgene was uniformly silenced and suggested the differences between their observation and those reported by Zhang et al. [19] may result from silencing a GFP transgene versus endogenous $p d s$. While a fluorescent tag can be used to localize the VIGS vector, the fluorescence signal does not reveal downstream biochemical effects occurring from gene silencing.

Raman and mass spectrometry (MS) imaging are complementary analysis techniques in regard to the nature of the signal, the kind of information measured, and the spatial resolution. Raman spectroscopy provides a fingerprint of functional groups within a molecule and is an ideal tool for carotenoid characterization. Raman scattering results when there is a change in photon energy as a result of exciting vibrations in chemical bonds. Resonance Raman spectroscopy (and pre-resonance Raman spectroscopy) occurs when the laser excitation wavelength falls within the range of wavelengths absorbed by a molecule and the result is an enhanced Raman scattering intensity. Raman imaging is a non-destructive technique that can be performed on whole tissue (such as fruit, leaves, stems, roots) or sections of these tissues and provides spatially-localized chemical information for a variety of compounds. The spatial distribution, relative content, and accumulation of carotenoids within plant tissues can be measured by plotting the area of a Raman peak to generate a Raman image [21-28]. These compounds, however, generally need to be abundant to be measured with Raman techniques. MS imaging is suitable to measure low abundance compounds with a greater chemical selectivity, and has become a valuable analytical

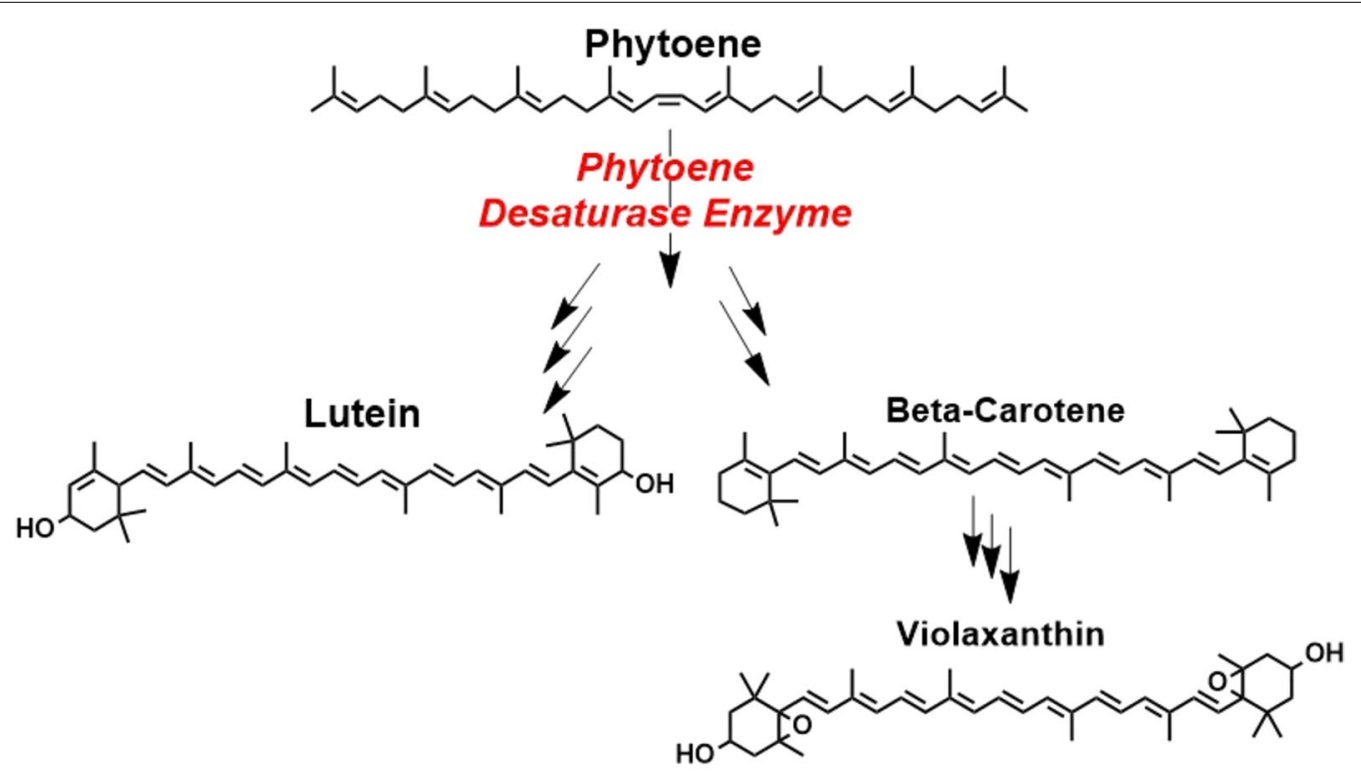

Fig. 1 Simplified carotenoid biosynthesis pathway in higher plants: phytoene (absorption and emission $\lambda, 291,360 \mathrm{~nm}$, respectively), $\beta$-carotene (absorption and emission $\lambda, 465,542 \mathrm{~nm}$, respectively), lutein (absorption and emission $\lambda, 456,525 \mathrm{~nm}$, respectively), and violaxanthin (absorption and emission $\lambda$ reported by Gruszecki et al. [57], 410, $555 \mathrm{~nm}$, respectively) 
tool for analyzing the spatial distribution of a wide range of compounds directly on or within plant tissues [29-32]. Matrix-assisted laser desorption/ionization (MALDI) imaging provides high sensitivity, and chemical versatility. Nanoparticles are efficient matrices for small molecule analysis using MALDI-MS imaging due to their low or negligible matrix background, homogeneous application, and high laser absorption [33]. In particular, silver nanoparticles have been commonly used for analyte cationization of various molecules, such as cholesterol, fatty acids, and other olefin-containing molecules [34-36].

Herein, a combined Raman and MS imaging approach is presented for the measurement of the cellular spatial dependence of $p d s$ silencing using the FoMV vector developed by Mei et al. [1] in the leaves of the maize sweet corn variety Golden $\times$ Bantam. The goal of this work is to gain a cellular level understanding of a VIGS phenotype using the downstream biochemical changes in carotenoid expression that occur from silencing $p d s$. We present a useful methodology suitable to study the spatial dependence of gene silencing at the cellular level using any VIGS virus targeting genes that produce a unique biochemical signature within leaf and stem tissues.

\section{Methods}

FoMV-pds silenced, FoMV, and non-inoculated sweet corn line golden $\mathrm{x}$ bantam plants

Plants were grown and inoculated as described by Mei et al. [1]. Plants were grown in a $20-22{ }^{\circ} \mathrm{C}$ greenhouse with a 16-h photoperiod. A Biolistic PDS-1000/He system was utilized to inoculate 1-week-old plants by particle bombardment with FoMV infectious clones (Bio-Rad Laboratories). The biolistic inoculation used $1 \mu \mathrm{m}$ gold particles coated with $1 \mu \mathrm{g}$ of FoMV plasmid DNA at 1100 p.s.i. to rupture disks from a distance of $6 \mathrm{~cm}$. Plants were placed in the dark for $12 \mathrm{~h}$ before and after bombardment with the FoMV infectious clones. The infected leaves were ground to sap with $50 \mathrm{mM}$ phosphate buffer $(\mathrm{pH} 7)$ and then rubbed onto plants with 600-mesh Carborundum at the two-leaf stage. The rub-inoculated plants were considered the "FoMV-pds silenced" plants. The "FoMV" plants were rub-inoculated with the FoMV vector without the sequence encoding the $p d s$ gene. "Non-Inoculated" plants were not inoculated with the FoMV vector. The following titles FoMV-pds, FoMV, and non-inoculated will be used throughout this work to describe the three plant types. Plants were approximately 5-6 weeks old or at the 5-9 leaf stage when measurements were completed. For all imaging experiments, the total leaf length was measured and the position representing half of the total leaf length was used for measurements. For full information about the VIGS system see Mei et al. [1]. Quantitative reverse transcription polymerase chain reaction (qRT-PCR) was used to determine the effectiveness within FoMV-pds silenced leaves. In leaves where the variegated phenotype was observed, there was a significant reduction (13.5-27.6\%) of $p d s$ expression when compared to the FoMV or non-inoculated leaves [1]. The VIGS methodology is also described in Mei et al. [37].

\section{Transverse cross sections of maize leaves}

A Leica CTI cryostat was utilized to section the leaves to $45 \mu \mathrm{m}$ thickness. Leaves 5 and 6 were manually cut with a scalpel into 5-8 cm samples at the half-leaf-length position. The FoMV-pds silenced leaves were sectioned at the highest abundance of variegated white areas at the half-leaf-length position. The cut samples were flash frozen in liquid nitrogen for $30 \mathrm{~s}$. The fixed tissues were placed vertically in the cryostat base molds which were filled halfway with distilled water. The samples were left in the cryostat set at $-23^{\circ} \mathrm{C}$ until the water was frozen. Sectioned samples were placed onto a microscope slide with a drop of distilled water, sealed with a glass coverslip and clear nail polish. Samples were stored in the dark and analyzed within $12 \mathrm{~h}$ of being sectioned.

\section{Fractured maize leaves}

Leaves were prepared as outlined previously by Klein et al. [38]. Leaves 5 and 6 were manually cut with a scalpel into $3-5 \mathrm{~cm}$ samples at the half-leaf-length position. The cut samples were placed on packing tape and vacuum dried. Once dried, the packing tape was folded over, passed through a rolling mill, and the two halves of the leaf were separated by pulling the tape apart.

\section{Mass spectrometry (MS) imaging}

The fractured maize leaves were subject to matrix deposition by sputter coating (108 Auto Sputter Coater, Ted Pella INC, Redding, CA, USA) silver at $40 \mathrm{~mA}$ for $10 \mathrm{~s}$. MS imaging data were collected using a MALDI-linear ion trap (LIT)-Orbitrap mass spectrometer (MALDILTQ-Orbitrap Discovery; Thermo Finnigan, San Jose, CA, USA). The instrument was modified to incorporate an external 355-nm frequency tripled Nd: YAG laser (UVFQ; Elforlight, Daventry, UK). TunePlus and XCalibur (ThermoFisher Scientific) were used to define imaging parameters and to acquire data, respectively. Mass spectra were acquired with 10 laser shots per spectrum in positive ion mode using an Orbitrap mass analyzer (resolution 30,000 at $\mathrm{m} / \mathrm{z} 400$ ) for an $\mathrm{m} / z$ scan range of $100-1000$ and using a $20 \mu \mathrm{m}$ raster step size. MS images were generated using ImageQuest (ThermoFisher Scientific) with a mass window of $\pm 0.003 \mathrm{Da}$. The imaged peak is a silver adduct, $\left[\mathrm{M}+{ }^{107} \mathrm{Ag}\right]^{+}$of phytoene. MS/ MS analysis was performed on a different region of the fractured leaf tissue to the one used for MS imaging. The 
ion-trap analyzer was used for MS/MS of selected ions with a mass window of $1.5 \mathrm{Da}$ and a normalized collision energy of 35 (arbitrary units). No downstream carotenoids were detected by MS imaging for any of the maize leaves using these experimental conditions suitable for detecting phytoene.

\section{Raman imaging}

Raman measurements were performed using a commercially available XploRA Plus Raman confocal upright microscope with a Synapse EMCCD camera (HORIBA Scientific, Edison, New Jersey). An Olympus objective $(20 \times$ magnification, 0.4 numerical aperture) was used to collect images in the epi-direction with a 1200 grooves/ millimeter grating and a $1450 \mathrm{~cm}^{-1}$ center wavelength, and a $100 \mu \mathrm{m}$ confocal pinhole. A 532-nm solid-state diode laser produced an $800 \mathrm{~W} / \mathrm{cm}^{2}$ laser irradiance, unless otherwise noted. A $75 \times 50 \mathrm{~mm}$ XYZ translational stage and a step size of $3 \mu \mathrm{m}$ was utilized for all Raman images. The size (and acquisition time) for the in situ whole leaf, leaf transverse cross sections, and fractured leaf Raman images were $50 \times 100 \mu \mathrm{m}(15 \mathrm{~s})$, $120 \times 238 \mu \mathrm{m}(5 \mathrm{~s})$, and $50 \times 50 \mu \mathrm{m}(15 \mathrm{~s})$, respectively. Two measurements per leaf and two separate leaves were measured for each plant type (i.e., FoMV-pds, FoMV, and non-inoculated). For the in situ whole leaf and leaf transverse cross sections. For the fractured leaf images, 2 areas on each side of the fractured leaf were collected for each plant type. White light optical images of the same area corresponding to the Raman images were collected using the same instrument. ImageJ was used to analyze optical images.

Igor Pro 6.36 (WaveMetrics, Inc., Lake Oswego, OR) scientific analysis and graphing software was used to process the Raman spectra. A Gaussian function with a constant baseline was used to batch fit and extract the $\sim 1520 \mathrm{~cm}^{-1}$ peak amplitudes and maxima. In order to generate Raman images, an automated method applied two criteria to the output of the resulting Gaussian fit functions: the peak maximum was between 1515 and $1530 \mathrm{~cm}^{-1}$, and the peak intensity had to be larger than three times the standard deviation of the noise. The noise was quantified within the region of $500-600 \mathrm{~cm}^{-1}$ where no spectral peaks are located. If any of the criteria were not met, the corresponding pixel was assigned null (a gray pixel) within the Raman image. The Raman images were plotted using a custom MATLAB 2016b script. All reported uncertainties represent one standard deviation.

Histograms of the $\sim 1520 \mathrm{~cm}^{-1}$ peak maximum were compiled for all spectra in four Raman images (in situ whole leaf and transverse leaf cross sections) or two images (fractured leaf). The bin width of the histogram was $1 \mathrm{~cm}^{-1}$, and the histogram was fit to a Gaussian function to obtain the reported distribution mean.

\section{Standard and supplement Raman measurements}

$\beta$-carotene (Sigma-Aldrich) and phytoene (Toronto Research Chemicals) standards were diluted to $0.01 \mathrm{mg} /$ $\mathrm{mL}$ in chloroform for absorbance and fluorescence measurements. Absorbance measurements were performed on an Agilent $8453 \mathrm{UV}$-visible spectrophotometer. Fluorescence measurements were carried out on an Agilent Cary Eclipse spectrophotometer. The excitation wavelength for fluorescence measurements was $291 \mathrm{~nm}$ for phytoene and $464 \mathrm{~nm}$ for $\beta$-carotene. For Raman measurements, the pure standards were diluted to $0.25 \mathrm{mg} /$ $\mathrm{mL}$ in chloroform and $3 \mu \mathrm{L}$ were drop casted (solvent evaporated) onto a clean (soaked in isopropanol and dried under a nitrogen flow) glass microscope cover slip. Raman spectra were collected with a $3 \mathrm{~s}$ acquisition, 2 accumulations and $1.66 \mathrm{~W} / \mathrm{cm}^{2}$ laser irradiance.

Lutein (Nature's Bounty®), zeaxanthin (Swanson Ultra $\odot$ ), lycopene (Spring Valley $\odot$ ), and $\beta$-carotene (Nature's Bounty $\odot$ ) liquid capsule dietary supplements were purchased from local retailers. Raman measurements required the contents of an individual capsule to be emptied onto a clean microscope slide. To collect the Raman spectrum of the supplement mixture, an equal volume of all the supplements was mixed together. The acquisition parameters were: $10 \mathrm{~s}$ acquisition, 2 accumulations and $1.66 \mathrm{~W} / \mathrm{cm}^{2}$ laser irradiance.

\section{Results}

\section{Carotenoid characterization by Raman spectroscopy}

Raman spectra of phytoene and $\beta$-carotene standards were measured to understand the Raman signal of these compounds and ultimately to enable the interpretation of the Raman images obtained from the maize leaves. The Raman spectrum of phytoene, when collected with a $532 \mathrm{~nm}$ laser, showed only a broad background and no Raman peaks (Fig. 2a), whereas the Raman spectrum of $\beta$-carotene showed peaks that are characteristic of carotenoids (Fig. 2b). There were three major vibrational modes $(v)$ in the Raman spectrum of carotenoids [22, 26, 39-42]. The most intense $v_{1}$ band corresponded to the inphase stretching vibrations of $\mathrm{C}=\mathrm{C}$ bonds, and occurred between 1512 and $1524 \mathrm{~cm}^{-1}$. The $v_{2}$ band corresponded to the stretching vibrations of $\mathrm{C}-\mathrm{C}$ bonds, and occurred at approximately $1150 \mathrm{~cm}^{-1}$. The lowest intensity $v_{3}$ band corresponded to the $\mathrm{C}-\mathrm{H}$ stretching modes, and occurred at approximately $1000 \mathrm{~cm}^{-1}$. The absorption spectra revealed the reason for the differences in the Raman spectra measured for phytoene and $\beta$-carotene. Phytoene does not absorb $532 \mathrm{~nm}$ light (Fig. 2c) and thus does not exhibit resonant enhancement of the Raman signal, 
a

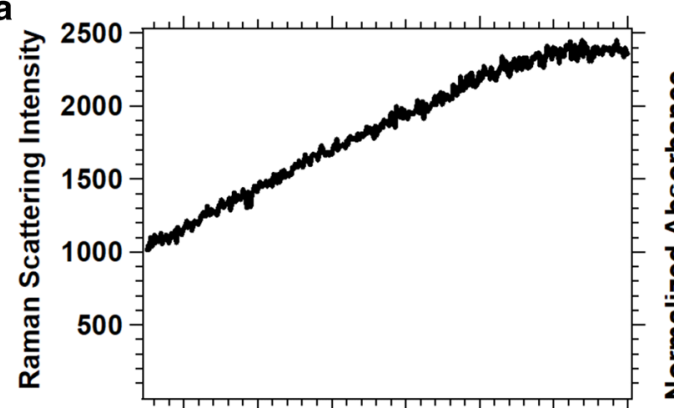

b

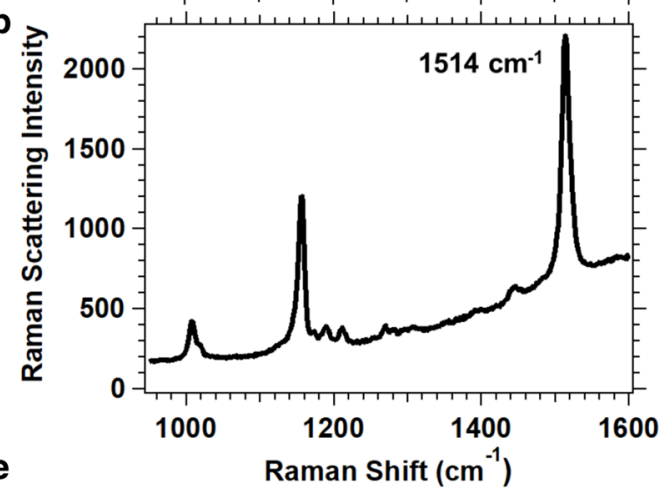

C

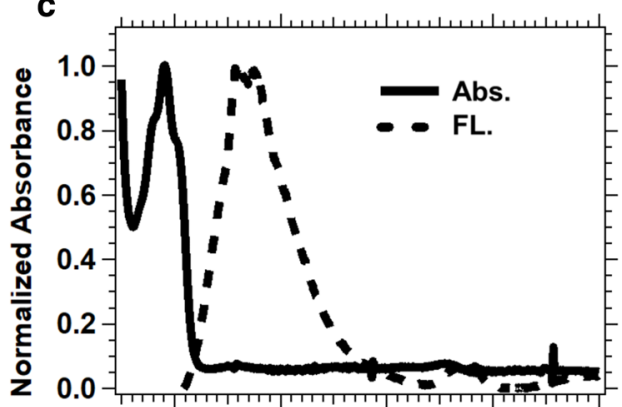

d

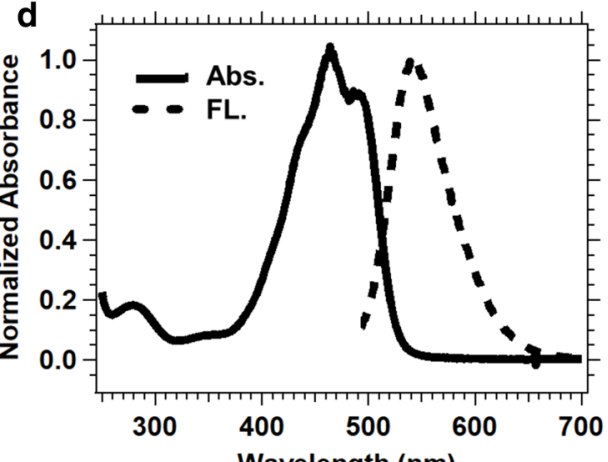

Wavelength (nm)

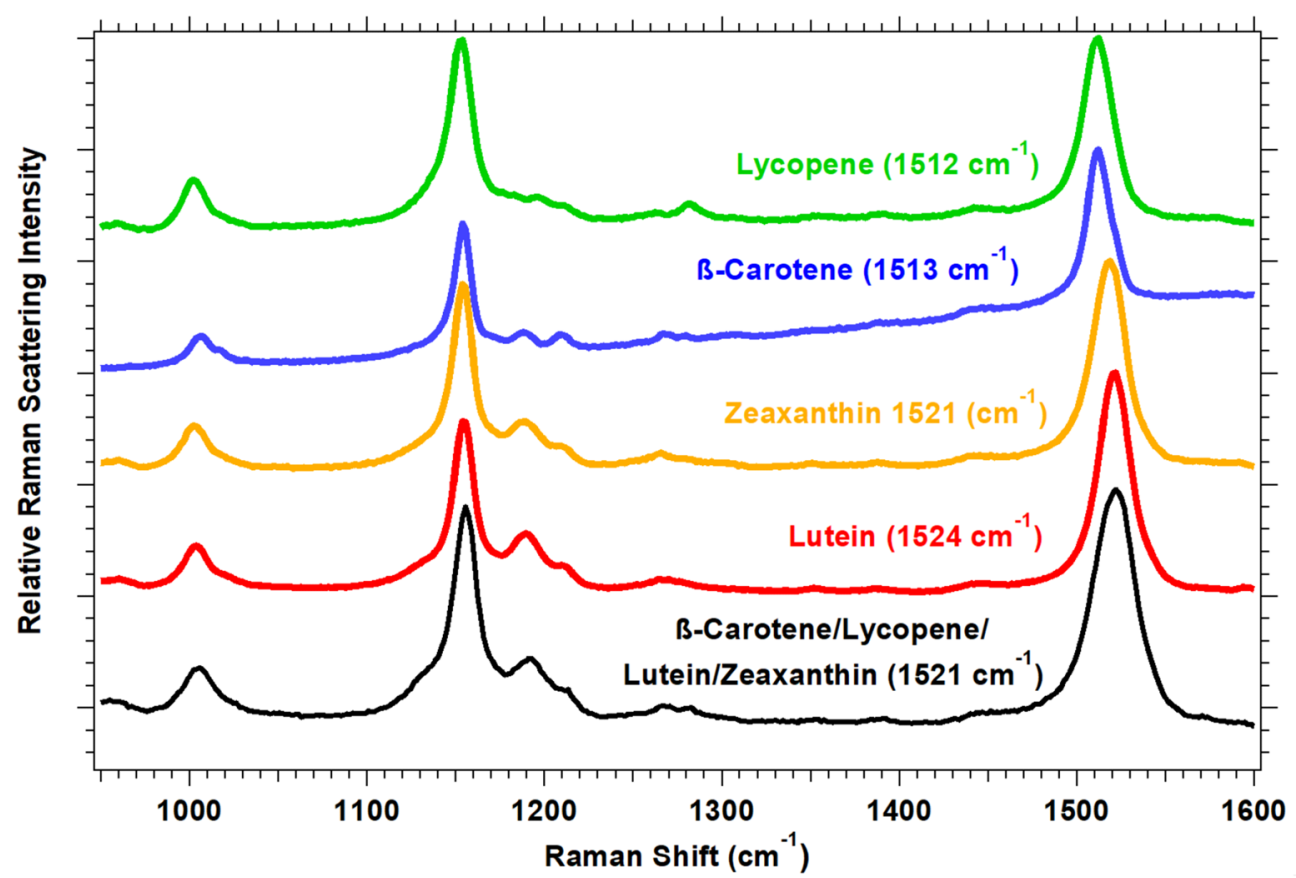

Fig. 2 Spectra of selected carotenoid $\mathbf{a}-\mathbf{d}$ standards and e supplements. a Raman, c absorption and fluorescence spectra of phytoene. b Raman, d absorption and fluorescence spectra of $\beta$-carotene. The phytoene absorption maximum is $291 \mathrm{~nm}$, which is not resonantly enhanced with a $532 \mathrm{~nm}$ laser, leading to a lack of peaks in the Raman spectrum. e Raman spectra of lycopene, $\beta$-carotene, zeaxanthin, lutein, and a mixture of the supplements with corresponding peak maxima

whereas $\beta$-carotene does absorb $532 \mathrm{~nm}$ light (Fig. 2d) and will exhibit an enhanced Raman signal, as will any carotenoid that absorbs the excitation wavelength.
Carotenoid supplements were utilized to determine how the Raman peaks were affected by their varying chemical structure (Fig. 2e). All the analyzed carotenoids absorbed $532 \mathrm{~nm}$ light to varying extents (Additional 
file 1: Fig. S1) and produced a strong Raman signal under these experimental conditions. There were few differences in the Raman peaks below $1450 \mathrm{~cm}^{-1}$. There were, however, differences in the $\sim 1520 \mathrm{~cm}^{-1}$ peak maximum, and these may allow changes in carotenoid mixtures to be identified by Raman spectroscopy. The $\sim 1520 \mathrm{~cm}^{-1}$ peak maximum depended on the degree of conjugation as well as the substituents on the tetraterpenoid backbone of the carotenoid. Lycopene and $\beta$-carotene possess 11 conjugated double bonds and no hydroxyl substituents and yielded a peak maximum at 1512 and $1513 \mathrm{~cm}^{-1}$ (Fig. 2e). The chemical structure of zeaxanthin and lutein have two hydroxyl substituents and the peak maximum was observed at 1521 and $1524 \mathrm{~cm}^{-1}$ (Fig. 2e). The spectral resolution of the Raman instrument determined whether the peak maxima can be distinguished. The instrument used to collect the Raman images in this work had a $5 \mathrm{~cm}^{-1}$ spectral resolution based on a spectral calibration with a Ne lamp. Thus, within the same spectrum $\beta$-carotene or lycopene peak maxima could be distinguished from lutein or zeaxanthin. A mixture of all four supplements had a peak maximum at $1521 \mathrm{~cm}^{-1}$ with a Gaussian peak shape and no evidence of spectrally overlapping peaks (Fig. 2e). For carotenoid mixtures, the peak maximum is expected to depend on the chemical composition and concentration of the constituents.

\section{Cellular spatial distribution of carotenoids: whole leaf measurements}

Overlays of the optical (white light illumination) and Raman images collected in situ on whole leaves are shown in Fig. 3a and Additional file 1: Fig. S2. The intensity of the $\sim 1520 \mathrm{~cm}^{-1}$ carotenoid peak was the largest and used to generate the Raman images to ensure the best sensitivity. The cell types visualized in the optical images were epidermal and guard cells. The white, primarily horizontal, stripes observed in all the optical images were only visible when the light was illuminating the leaf on the same side as the detector (epi-illumination) as opposed to when the illumination and detector were on opposite sides (trans-illumination) (Additional file 1: Fig. S4). This indicated the striped features were

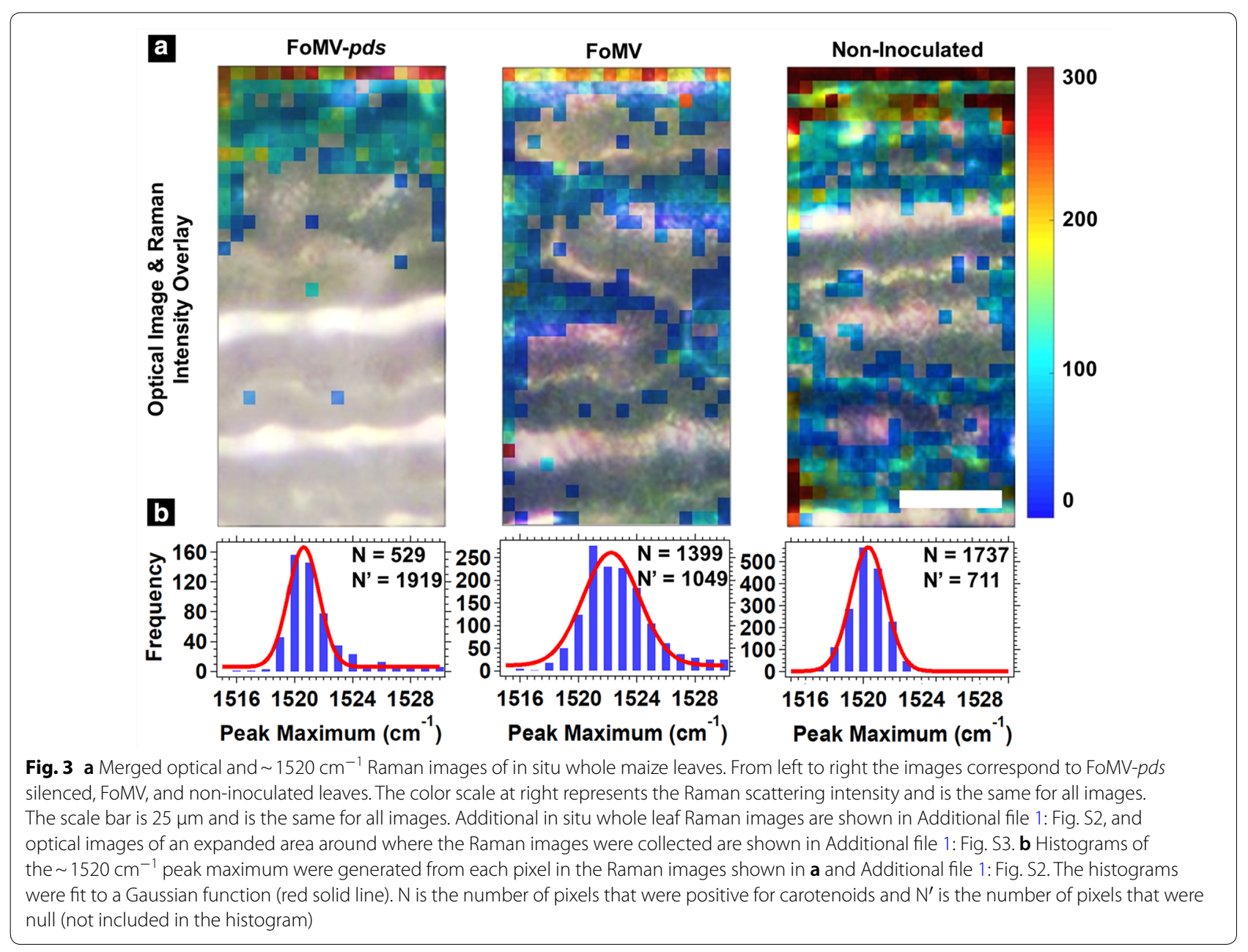


generated from light reflection or scattering from the exterior (cuticle) layer of the leaf. A representative camera image of the FoMV-pds, FoMV, and non-inoculated leaves with $p d s$ silenced areas outlined within the FoMV$p d s$ plant type is shown within Additional file 1: Fig. S5). Within the FoMV-pds silenced leaf, the carotenoid signal was not measured in most of the pixels at the bottom of the image where the FoMV mosaic pattern was visually observed (Fig. 3a). (Optical images of the areas adjacent to where the Raman images were collected are shown in Additional file 1: Fig. S3). This indicated a reduction in carotenoid expression within these measured areas. The few pixels that showed a carotenoid signal in the variegated white phenotype area were located where the scattering/reflection of light at the leaf surface was the lowest and may represent areas where the excitation light penetrates farther into the plant tissue. For the FoMV and non-inoculated plants (Fig. 3a), the carotenoid signals were distributed more evenly throughout the Raman image. The peak maximum histograms for the FoMV-pds silenced, FoMV, and non-inoculated leaves (Fig. 3b) had a mean ranging from 1521 to $1522 \mathrm{~cm}^{-1}$, suggesting when carotenoids were present there was a similar carotenoid composition in all plant types.

\section{Cellular spatial distribution of carotenoids: transverse leaf cross section measurements}

As mentioned above, the excitation laser may have probed through different tissue depths at different locations on the whole leaf, and the in situ whole leaf measurements were not ideal for obtaining information about internal cellular structures including the mesophyll and vascular bundles. Raman images of transverse cross sections of the leaf revealed the internal cellular structures (Fig. 4). Each image showed at least two vascular bundles with the surrounding mesophyll, and epidermal cells present on the left and right of the vascular bundles (Fig. 4a). The FoMV and non-inoculated optical and Raman images of the transverse cross sections showed a correlation between chloroplast location and carotenoid signal. The correlation of chloroplast location and carotenoid signal were in agreement with the location

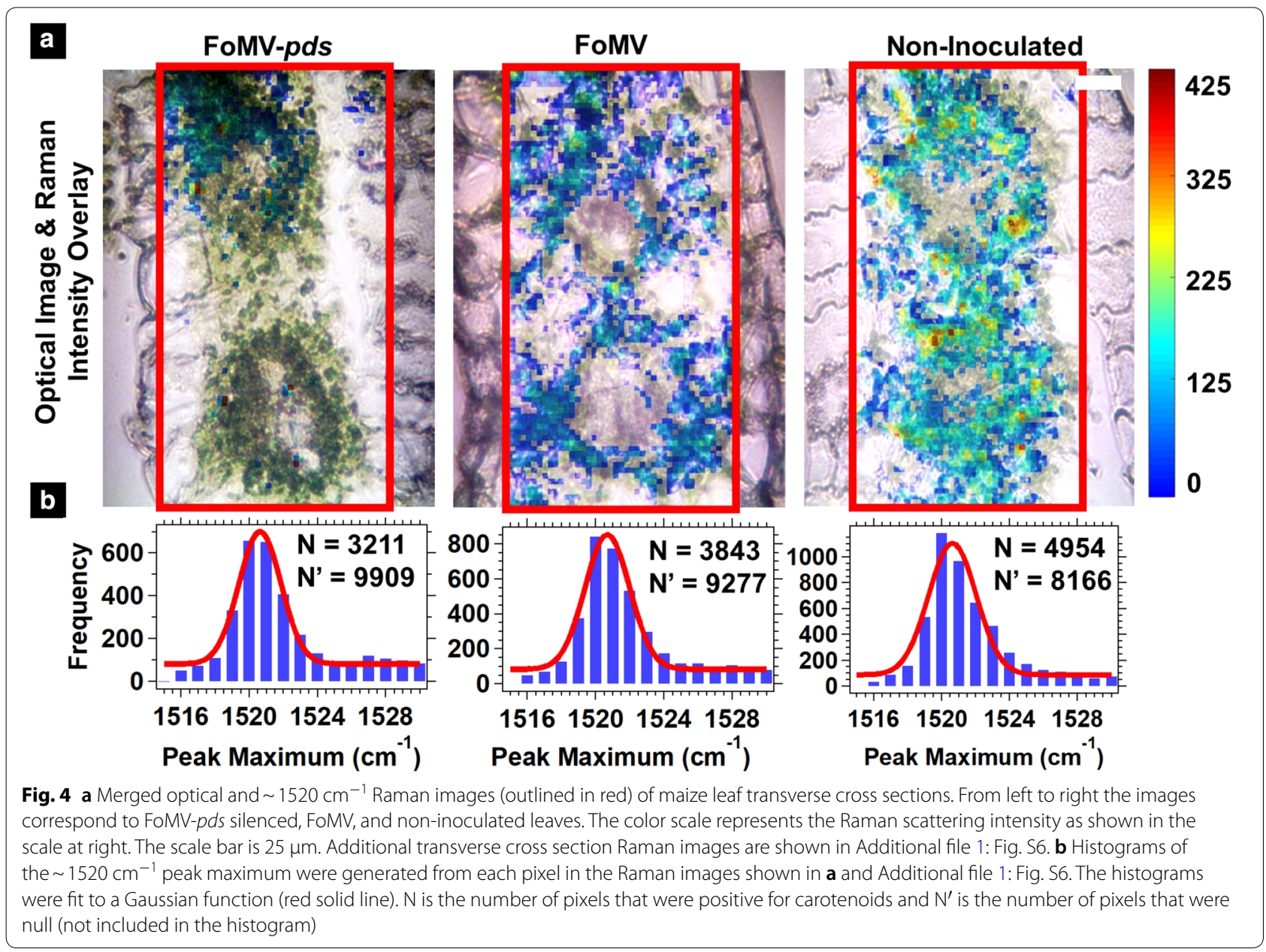


of carotenoid biosynthesis [43]. The overall carotenoid signal from replicates of the same plant type (FoMV$p d s$, FoMV, and non-inoculated) (Additional file 1: Fig. S6), showed extreme variability, for this reason no conclusions about differences in the spatial location of the carotenoid signal among the three plant types were made from data collected with this sample preparation method. The $\sim 1520 \mathrm{~cm}^{-1}$ peak maximum histograms generated from the transverse cross section data (Fig. 4b) were consistent with the values obtained for the in situ whole leaf measurements (Fig. 3b) with a distribution maximum between 1521 and $1522 \mathrm{~cm}^{-1}$, indicating a similar carotenoid composition is measured in both sample preparation methods.

\section{Complementary Raman and mass spectrometry imaging of fractured maize leaves}

Fracturing the leaves provided a way to consistently correlate the Raman signal from internal leaf structures and the visible phenotype observed from the exterior of the leaf, although some cellular structures may have been altered from the fracturing process (Fig. 5). Raman images collected on the variegated white areas of the FoMV-pds silenced fractured leaves showed low, but not zero, levels of carotenoid Raman signal (Fig. 5a); whereas the green areas (Fig. 5b) showed carotenoid signals consistent with the FoMV (Fig. 5c) and non-inoculated (Fig. 5d) fractured leaves with a moderately uniform carotenoid signal. The $\sim 1520 \mathrm{~cm}^{-1}$ peak maximum histogram mean ranged from 1523 to $1525 \mathrm{~cm}^{-1}$ for the FoMV-pds silenced, FoMV, and non-inoculated fractured leaves.

Phytoene did not produce an appreciable Raman signal with the experimental conditions used for this study, therefore MS imaging was used to detect phytoene and confirm the spatial localization within the fractured leaves. A sputter coated silver matrix was used for detection. The $m / z$ assignment $\left(m / z\right.$ 651.405, $\left.\left[\mathrm{M}+{ }^{107} \mathrm{Ag}\right]^{+}\right)$ was based on accurate mass measurement, and confirmed by MS/MS [44] (Additional file 1: Fig. S7). Low phytoene signal was present in the MS image of the FoMV and non-inoculated fractured leaf, indicating there was no appreciable buildup of phytoene (Fig. 6). On the contrary, there was a specific localization of phytoene signal within the FoMV-pds silenced fractured leaf originating from the accumulation of phytoene.

High performance liquid chromatography (HPLC) was performed on the leaf samples to quantify the concentration of selected carotenoids (Additional file 1: Table S1). The concentration of phytoene extracted from the FoMV$p d s$ leaf and analyzed by HPLC was $0.4 \pm 0.2 \mu \mathrm{g} / \mathrm{g}$ of fresh weight (see Additional file 1 for extraction method). Phytoene was not detected by HPLC in the extract of the

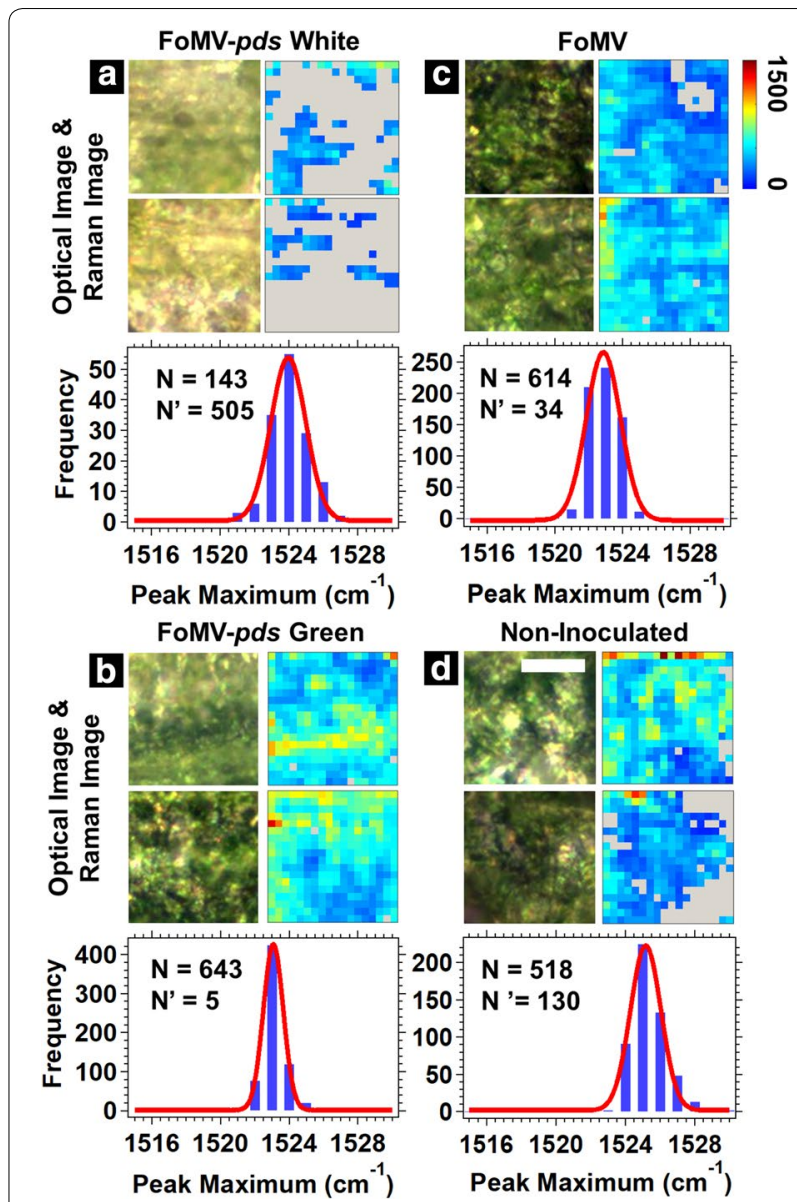

Fig. 5 a-d Optical images, $\sim 1520 \mathrm{~cm}^{-1}$ Raman images of fractured maize leaves, and histograms of the $1520 \mathrm{~cm}^{-1}$ peak location generated from each pixel within the Raman images. The fracturing process splits the leaf into two halves lengthwise. For each panel, the top optical and Raman image corresponds to one side of the fractured leaf, and the bottom optical and Raman image corresponds to the other side of the fractured leaf. Two areas at the half-leaf-length position were analyzed for the FoMV-pds leaf: a variegated white area and $\mathbf{b}$ a green area. The scale bar is $25 \mu \mathrm{m}$. The histograms were fit to a Gaussian function (red solid line). $\mathbf{c}, \mathbf{d}$ represent the area measured for the FoMV and non-inoculated fractured leaves, respectively. $\mathrm{N}$ is the number of pixels that were positive for carotenoids and $\mathrm{N}^{\prime}$ is the number of pixels that were null (colored gray and were not included in the histogram)

non-inoculated leaves and was measured at a very low concentration $0.011 \pm 0.005 \mu \mathrm{g} / \mathrm{g}$ of fresh weight within two of the replicates for the FoMV leaf. (The HPLC limit of detection for phytoene was $0.003 \mu \mathrm{g} / \mathrm{g}$ of fresh weight.) The quantification performed with the extract was consistent with the MS images that showed a very low phytoene signal across the FoMV and non-inoculated leaf. The downstream carotenoids (Fig. 1) measured in the non-inoculated leaf were: lutein $(61 \pm 3 \mu \mathrm{g} / \mathrm{g}), \alpha$ and $\beta$-carotene $(22 \pm 1 \mu \mathrm{g} / \mathrm{g})$, violaxanthin/neoxanthin 


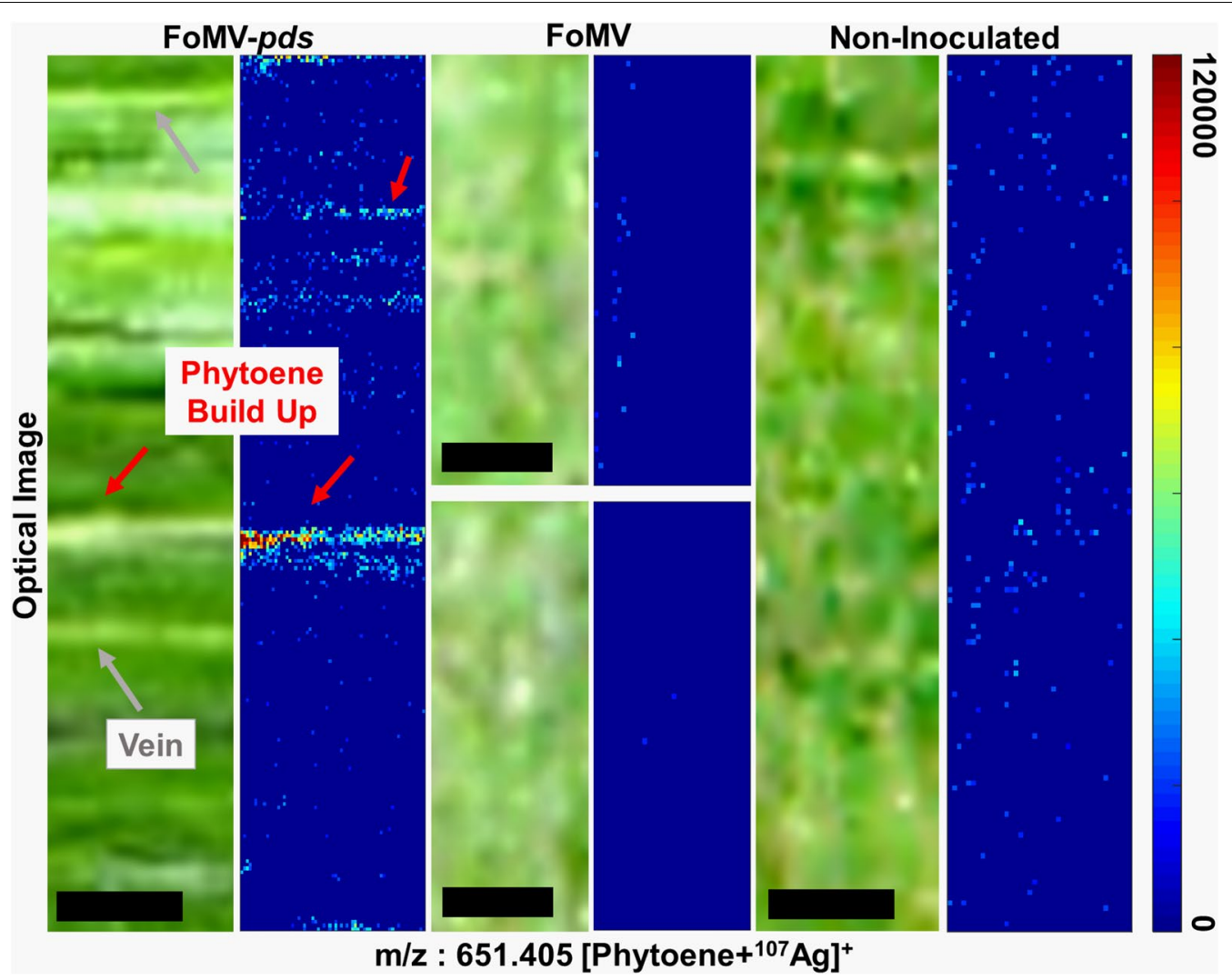

Fig. 6 Mass spectrometry (MS) images of fractured FoMV-pds silenced, FoMV and non-inoculated maize leaves. The images are generated using the phytoene mass-to-charge $(\mathrm{m} / \mathrm{z})$ ratio 651.405 , which is a silver adduct [Phytoene $\left.+{ }^{107} \mathrm{Ag}\right]{ }^{+}$. The ion intensity is shown using the color scale shown on the right. The leaf vein(s) were identifiable (gray arrows) because they were present along the entire length of the leaf. The FoMV-pds silenced variegated white areas (red arrows) however were not present along the entire length of the leaf. The resolution of the camera collecting the optical images on the MSI instrument is low, however an additional optical image collected prior to imaging is provided in Additional file 1: Fig. S8. Scale bar is $500 \mu \mathrm{m}$ for all images

$(10 \pm 2 \mu \mathrm{g} / \mathrm{g})$, and zeaxanthin $(7 \pm 5 \mu \mathrm{g} / \mathrm{g})$. Comparing the FoMV leaf to the non-inoculated leaf, the only statistically significant difference $(p<0.05)$ was a lower concentration of $\alpha$ and $\beta$-carotene within the FoMV leaf. Comparing the FoMV-pds leaf to the non-inoculated leaf there were no statistically significant differences in the downstream carotenoid concentrations. This observation justifies a need for chemical imaging to provide biochemical information at specific spatially-correlated locations to measure changes in the local carotenoid expression that were lost in the spatially-averaged signal that is obtained from the extract analysis.

\section{Discussion}

The most common method used to analyze the effectiveness of VIGS is qRT-PCR [1,9]. The mRNA transcript levels are generally measured using a large amount of tissue (i.e., spatially averaged) and are reported as a percentage change relative to non-inoculated tissue. This technique is not able to reveal downstream biochemical effects occuring as a result of VIGS at the cellular level. While visual inspection of the plant tissue can reveal the macroscopic variegation that results from $p d s$ silencing, such a simple analysis cannot reveal details at the cellular level, provide information about specific biochemical compounds, nor easily differentiate varying low levels of response. FoMV-pds suppresses expression of the mRNA transcripts encoding the $p d s$ enzyme and this is expected to result in the accumulation of the colorless phytoene in leaves $[11,45]$. The accumulation of phytoene cannot be detected by a visible phenotype. The analysis of the extracted carotenoids demonstrates an increase in phytoene within the FoMV-pds silenced leaf. MS imaging revealed an increased phytoene signal that was spatially localized within the variegated areas of the fractured leaves of the FoMV-pds silenced leaves (Fig. 6). This agrees with the reduced carotenoid Raman signal within the same area (Fig. 5). The FoMV and 
non-inoculated fractured leaf MS images display a low non-localized phytoene signal. Even though $p d s$ silencing does not result in the complete elimination of the mRNA expression within FoMV-pds tissues [1], the multimodal imaging technique still measures the biochemical changes in carotenoid expression that occurred from the achieved level of gene silencing. The measurable changes are expected to be easier to detect by the outlined imaging approach when the silencing levels are higher.

The carotenoid extract shows no statistically significant differences in any of the carotenoids measured by HPLC when comparing the non-inoculated and FoMV$p d s$ leaves. This is not surprising when considering the mosaic pattern of infection and $p d s$ silencing only affects a small area of the overall leaf. It is difficult to quantify small differences in the bulk of the extracted components. Imaging approaches, on the other hand, allow for a localized distribution to be measured and reveal spatially-correlated differences lost in the average when analyzing an extraction. The percentage of pixels that have a detectable carotenoid signal within the Raman images for the FoMV-pds silenced in situ whole leaves is $22 \pm 5 \%(n=4)$ in the areas measured. The percentage of pixels that have a detectable carotenoid signal for FoMV and non-inoculated in situ whole leaves are $57 \pm 9 \%$ and $70 \pm 10 \%$, respectively. The fractured leaf images reveal similar results. The percentage of pixels that have a detectable carotenoid signal for FoMV-pds silenced, FoMV, and non-inoculated fractured leaves are $22 \pm 2 \%(n=2), 99.1 \pm 0.3 \%, 80 \pm 8 \%$, respectively. Within the region that produces a visible mosaic phenotype, the carotenoid expression is reduced but not eliminated and is most evident in the fractured leaf images.

The most abundant downstream carotenoids reported in the literature for the leaves of maize and other monocots are lutein, $\beta$-carotene, and violaxanthin [46-50]. The extracted carotenoids measured by HPLC are (in order of abundance): lutein, $\alpha$ and $\beta$-carotene, violaxanthin/neoxanthin, and zeaxanthin. Other carotenoids may also be present as a comprehensive HPLC analysis has not been performed. The histograms of the $\sim 1520 \mathrm{~cm}^{-1}$ Raman peak maximum provide information about the nature of the carotenoids present. The $1521-1522 \mathrm{~cm}^{-1}$ peak maximum measured for the in situ whole leaf and transverse cross section is consistent with a mixture consisting of primarily lutein and $\beta$-carotene. The fractured leaves have a $1-5 \mathrm{~cm}^{-1}$ higher mean peak maximum compared to the in situ whole leaf and transverse cross section measurements, which is not likely the result of biologically relevant differences in chemical compositions since the same samples were analyzed for the three leaf preparation methods. Carotenoids are susceptible to oxidation, especially if the plant is under stressors including excess light or dehydration [51-55]. The preparation of a fractured leaf requires dehydration, unlike the other two sample preparation methods, and oxidation of the carotenoids upon fracturing the leaf may explain the shift in the peak maximum to higher wavenumbers.

Raman and MS imaging are complementary imaging approaches in many aspects: (i) non-destructive versus destructive sampling; (ii) subcellular resolution of $\sim 1 \mu \mathrm{m}$ versus a cellular resolution of $\sim 10 \mu \mathrm{m}$; (iii) knowledge of functional groups present versus the mass of the molecules present; and (iv) molecular sensitivity to resonance with the excitation laser for Raman imaging versus a high ionization efficiency with MS imaging. Whereas the differential sensitivity of MS imaging enables the imaging of phytoene, the precursor to downstream carotenoids, Raman imaging provides high selectivity for downstream carotenoids.

\section{Conclusion}

In summary, a multimodal Raman and MS imaging method has been successfully demonstrated in the current study to measure the spatial dependence of biochemical changes from gene silencing at the cellular level. This information is vital in gaining a complete understanding of the loss-of function phenotypes produced by VIGS. The most common analysis of VIGS efficacy provides an average percentage change in the mRNA levels with no spatial correlation of biochemical changes at the cellular level. We report in regions of the leaf that show a visible mosaic phenotype, the carotenoid expression is reduced but not completely eliminated. The presented multimodal imaging approach will be useful to study the silencing of plant genes that produce a unique biochemical signature even if the gene silencing does not produce a visible phenotype. If the phenotype is not visible, it is advisable to sample multiple locations, possibly multiple tissues, to ensure heterogeneous biochemical responses are measured. We have measured up to six locations on the same leaf (data not shown) without observing any signal degradation, and more measurements should be possible on the same leaf. This proof-of-concept study used the common VIGS marker gene, $p d s$, which is easily detected by the characteristic variegated phenotype. We propose this method should be used in conjunction with gene expression analysis (e.g., qRT-PCR) to confirm that gene silencing was successful. The imaging approach can then be applied to measure resulting biochemical changes that result from the reduced gene expression. For example, the resulting biochemical changes may be measured for plant genes that alter the synthesis of lignin, cellulose, or carotenoids. Raman and MS imaging [56] can provide spatially-correlated biochemical 
compositions in the leaf, and stem of monocots (Additional file 1: Fig. S9) and dicots (Additional file 1: Fig. S10). In addition, the presented approach will also be useful for measuring biochemical changes that result, for example, from biotic and abiotic stresses.

\section{Additional file}

Additional file 1. Additional materials and methods, images, and spectra.

\section{Abbreviations}

pds: phytoene desaturase; FoMV: Foxtail mosaic virus; VIGS: virus-induced gene silencing; ORFs: open reading frames; GFP: green fluorescent protein; MS: mass spectrometry; MALDI: matrix-assisted laser desorption/ionization; HPLC: high performance liquid chromatography.

\section{Authors' contributions}

EAS and SAW conceived the project and research plans; EAS, SAW, BJN, and YJL supervised the experiments; SJB, NMS, YM, MED, DJF, and GD designed and performed the experiments and analyzed the data; SCS provided technical assistance to SJB; SJB wrote the article with contributions of all the authors; EAS and SAW supervised and complemented the writing. All authors read and approved the final manuscript.

\section{Author details}

${ }^{1}$ The Ames Laboratory, U.S. Department of Energy, lowa State University, Ames, IA 50011-3111, USA. ${ }^{2}$ Department of Chemistry, lowa State University, Ames, IA 50011-3111, USA. ${ }^{3}$ Department of Plant Pathology and Microbiology, lowa State University, Ames, IA 50011, USA. ${ }^{4}$ Department of Biochemistry Biophysics, and Molecular Biology, Center for Metabolic Biology, lowa State University, Ames, IA 50011, USA. ${ }^{5}$ Engineering Research Center for Biorenewable Chemicals, lowa State University, Ames, IA 50011, USA.

\section{Acknowledgements}

The WM Keck Metabolomics Research Laboratory (lowa State University, Ames, IA) is acknowledged for providing access to instrumentation and discussion of the HPLC analyses.

\section{Competing interests}

The authors declare that they have no competing interests.

\section{Availability of data and materials}

The datasets used and/or analyzed during the current study are available from the corresponding author on reasonable request.

\section{Consent for publication}

Not Applicable.

\section{Ethics approval and consent to participate}

Maize plants used in the study were modified and grown within lowa State University greenhouses and experiments complied with local and national guidelines.

\section{Funding}

SJB, NMS, DJF and EAS are supported by the U.S. Department of Energy, Office of Science, Office of Biological and Environmental Research (BER) through the Ames Laboratory. The Ames Laboratory is operated for the U.S. Department of Energy by lowa State University under Contract No. DE-AC02-07CH1 1358. MED, GD, YJL, and BJN are supported by BER through award DESC0014038 to lowa State University. YM and SAW are supported by the lowa State University Plant Sciences Institute and the USDA NIFA Hatch Project No. 3808.

\section{Publisher's Note}

Springer Nature remains neutral with regard to jurisdictional claims in published maps and institutional affiliations.
Received: 5 January 2018 Accepted: 12 May 2018

Published online: 25 May 2018

\section{References}

1. Mei Y, Zhang C, Kernodle BM, Hill JH, Whitham SA. A Foxtail mosaic virus vector for virus-induced gene silencing in maize. Plant Physiol. 2016;171:760-72

2. Liu N, Xie K, Jia Q, Zhao J, Chen T, Li H, Wei X, Diao X, Hong Y, Liu Y. Foxtail mosaic virus-induced gene silencing in monocot plants. Plant Physiol. 2016;171:1801-7.

3. Liou M-R, Huang Y-W, Hu C-C, Lin N-S, Hsu Y-H. A dual gene-silencing vector system for monocot and dicot plants. Plant Biotechnol J. 2014;12:330-43.

4. Liu Z, Kearney CM. An efficient Foxtail mosaic virus vector system with reduced environmental risk. BMC Biotechnol. 2010;10:88.

5. Scofield SR, Huang L, Brandt AS, Gill BS. Development of a virus-induced gene-silencing system for hexaploid wheat and its use in functional analysis of the Lr21-mediated leaf rust resistance pathway. Plant Physiol. 2005;138:2165-73.

6. Lu H-C, Chen H-H, Tsai W-C, Chen W-H, Su H-J, Chang DC-N, Yeh H-H. Strategies for functional validation of genes involved in reproductive stages of orchids. Plant Physiol. 2007;143:558-69.

7. Ding XS, Schneider WL, Chaluvadi SR, Mian MAR, Nelson RS. Characterization of a brome mosaic virus strain and its use as a vector for gene silencing in monocotyledonous hosts. Mol Plant Microbe Interact. 2006;19:1229-39.

8. Purkayastha A, Mathur S, Verma V, Sharma S, Dasgupta I. Virus-induced gene silencing in rice using a vector derived from a DNA virus. Planta. 2010;232:1531-40.

9. Wang R, Yang X, Wang N, Liu X, Nelson RS, Li W, Fan Z, Zhou T. An efficient virus-induced gene silencing vector for maize functional genomics research. Plant J. 2016;86:102-15.

10. Bruun-Rasmussen M, Madsen CT, Johansen E, Albrechtsen M. Revised sequence of foxtail mosaic virus reveals a triple gene block structure similar to potato virus X. Adv Virol. 2008;153:223-6.

11. Kumagai MH, Donson J, Della-Cioppa G, Harvey D, Hanley K, Grill LK. Cytoplasmic inhibition of carotenoid biosynthesis with virus-derived RNA. Proc Natl Acad Sci USA. 1995;92:1679-83.

12. Robertson NL, French R, Morris TJ. The open reading frame $5 A$ of foxtail mosaic virus is expressed in vivo and is dispensable for systemic infection. Adv Virol. 2000;145:1685-98.

13. Hannoufa A, Hossain Z. Regulation of carotenoid accumulation in plants. Biocatal Agric Biotechnol. 2012;1:198-202.

14. Wurtzel ET, Cuttriss A, Vallabhaneni R. Maize provitamin A carotenoids, current resources, and future metabolic engineering challenges. Front Plant Sci. 2012;3:29.

15. Bartley GE, Scolnik PA. Plant carotenoids: pigments for photoprotection, visual attraction, and human health. Plant Cell. 1995;7:1027-38.

16. Bartley GE, Scolnik PA, Giuliano G. Molecular biology of carotenoid biosynthesis in plants. Annu Rev Plant Physiol Plant Mol Biol. 1994:45:287-301.

17. Beyer $\mathrm{P}$, Mayer $\mathrm{M}$, Kleinig $\mathrm{H}$. Molecular oxygen and the state of geometric isomerism of intermediates are essential in the carotene desaturation and cyclization reactions in daffodil chromoplasts. Eur J Biochem. 1989;184:141-50.

18. Chen Y, Li F, Wurtzel ET. Isolation and characterization of the Z-ISO gene encoding a missing component of carotenoid biosynthesis in plants. Plant Physiol. 2010;153:66-79.

19. Zhang C, Bradshaw JD, Whitham SA, Hill JH. The development of an efficient multipurpose bean pod mottle virus viral vector set for foreign gene expression and RNA silencing. Plant Physiol. 2010;153:52.

20. Juvale PS, Hewezi T, Zhang C, Kandoth PK, Mitchum MG, Hill JH, Whitham SA, Baum TJ. Temporal and spatial Bean pod mottle virus-induced gene silencing in soybean. Mol Plant Pathol. 2012;13:1140-8.

21. Gierlinger N, Schwanninger M. The potential of Raman microscopy and Raman imaging in plant research. Spectroscopy. 2007;21:69-89.

22. Schulz H, Baranska M, Baranski R. Potential of NIR-FT-Raman spectroscopy in natural carotenoid analysis. Biopolymers. 2005;77:212-21. 
23. Baranska M, Schulz H, Rosch P, Strehle MA, Popp J. Identification of secondary metabolites in medicinal and spice plants by NIR-FT-Raman microspectroscopic mapping. Analyst. 2004;129:926-30.

24. Baranski R, Baranska M, Schulz H. Changes in carotenoid content and distribution in living plant tissue can be observed and mapped in situ using NIR-FT-Raman spectroscopy. Planta. 2005;222:448-57.

25. Weissflog I, Vogler N, Akimov D, Dellith A, Schachtschabel D, Svatos A, Boland W, Dietzek B, Popp J. Toward in vivo chemical imaging of epicuticular waxes. Plant Physiol. 2010;154:604-10.

26. Baranska M, Roman M, Dobrowolski J, Schulz H, Baranski R. Recent advances in Raman analysis of plants: alkaloids, carotenoids, and polyacetylenes. Curr Anal Chem. 2013;9:108-27.

27. Strehle MA, Rösch P, Baranska M, Schulz H, Popp J. On the way to a quality control of the essential oil of fennel by means of Raman spectroscopy. Biopolymers. 2005;77:44-52.

28. Roman M, Marzec KM, Grzebelus E, Simon PW, Baranska M, Baranski R. Composition and (in)homogeneity of carotenoid crystals in carrot cells revealed by high resolution Raman imaging. Spectrochim Acta Part A Mol Biomol Spectrosc. 2015;136, Part C:1395-400.

29. Dueñas ME, Klein AT, Alexander LE, Yandeau-Nelson MD, Nikolau BJ, Lee YJ. High-spatial resolution mass spectrometry imaging reveals the genetically programmed, developmental modification of the distribution of thylakoid membrane lipids among individual cells of the maize leaf. Plant J. 2017;89:825-38.

30. Sturtevant D, Lee YJ, Chapman KD. Matrix assisted laser desorption/ionization-mass spectrometry imaging (MALDI-MSI) for direct visualization of plant metabolites in situ. Curr Opin Biotechnol. 2016;37:53-60.

31. Lee YJ, Perdian DC, Song Z, Yeung ES, Nikolau BJ. Use of mass spectrometry for imaging metabolites in plants. Plant J. 2012;70:81-95.

32. Feenstra AD, Alexander LE, Song Z, Korte AR, Yandeau-Nelson M, Nikolau BJ, Lee YJ. Spatial mapping and profiling of metabolite distributions during germination. Plant Physiol. 2017. https://doi.org/10.1104/pp.17.00652.

33. Chiang C-K, Chen W-T, Chang H-T. Nanoparticle-based mass spectrometry for the analysis of biomolecules. Chem Soc Rev. 2011;40:1269-81.

34. Dufresne M, Thomas A, Breault-Turcot J, Masson J-F, Chaurand P. Silverassisted laser desorption ionization for high spatial resolution imaging mass spectrometry of olefins from thin tissue sections. Anal Chem. 2013:85:3318-24

35. Jun JH, Song Z, Liu Z, Nikolau BJ, Yeung ES, Lee YJ. High-spatial and highmass resolution imaging of surface metabolites of arabidopsis thaliana by laser desorption-ionization mass spectrometry using colloidal silver. Anal Chem. 2010:82:3255-65.

36. Sekula J, Niziol J, Rode W, Ruman T. Silver nanostructures in laser desorption/ionization mass spectrometry and mass spectrometry imaging. Analyst. 2015;140:6195-209.

37. Mei Y, Whitham SA. Virus-induced gene silencing in maize with a Foxtail mosaic virus vector. In: Lagrimini LM, editor. Maize: methods and protocols. New York: Springer; 2018. p. 129-39.

38. Klein AT, Yagnik GB, Hohenstein JD, Ji Z, Zi J, Reichert MD, Maclntosh GC, Yang B, Peters RJ, Vela J, et al. Investigation of the chemical interface in the soybean-aphid and rice-bacteria interactions using MALDI-mass spectrometry imaging. Anal Chem. 2015;87:5294-301.
39. Pascal AA, Ruban AV, Robert B. Antenna protein conformational changes revealed by resonance raman spectroscopy. In: Demmig-Adams B, Garab G, Adams lii W, Govindjee Ul, editors. Non-photochemical quenching and energy dissipation in plants, algae and cyanobacteria. Dordrecht: Springer; 2014. p. 245-57.

40. Ermakov IV, Sharifzadeh M, Ermakova M, Gellermann W. Resonance Raman detection of carotenoid antioxidants in living human tissue. J Biomed Opt. 2005;10:064028.

41. Withnall R, Chowdhry BZ, Silver J, Edwards HGM, de Oliveira LFC. Raman spectra of carotenoids in natural products. Spectrochim Acta Part A Mol Biomol Spectrosc. 2003;59:2207-12.

42. Ladislav F, Pacakova V, Stulik K, Volka K. Reliability of carotenoid analyses: a review. Curr Anal Chem. 2005;1:93-102.

43. Joyard J, Ferro M, Masselon C, Seigneurin-Berny D, Salvi D, Garin J, Rolland N. Chloroplast proteomics and the compartmentation of plastidial isoprenoid biosynthetic pathways. Mol Plant. 2009;2:1154-80.

44. Sherrod SD, Diaz AJ, Russell WK, Cremer PS, Russell DH. Silver nanoparticles as selective ionization probes for analysis of olefins by mass spectrometry. Anal Chem. 2008;80:6796-9.

45. Liu Y, Schiff M, Dinesh-Kumar SP. Virus-induced gene silencing in tomato. Plant J. 2002;31:777-86

46. Wolf FT. Effects of light and darkness on biosynthesis of carotenoid pigments in wheat seedlings. Plant Physiol. 1963;38:649-52.

47. Kay RE, Phinney B. Plastid pigment changes in the early seedling leaves of Zea Mays L. Plant Physiol. 1956;31:226-31.

48. Kay RE, Phinney BO. The control of plastid pigment formation by a virescent gene, pale-yellow-1, of maize. Plant Physiol. 1956;31:415-20.

49. Arvayo-Enriquez H, Mondaca-Fernandez I, Gortarez-Moroyoqui P, LopezCervantes J, Rodriguez-Ramirez R. Carotenoids extraction and quantification: a review. Anal Methods. 2013;5:2916-24.

50. Takagi S. Determination of green leaf carotenoids by HPLC. Agric Biol Chem. 1985;49:1211-3.

51. Merzlyak MN, Gitelson AA, Pogosyan SI, Lekhimena L, Chivkunova OB. Light-induced pigment degradation in leaves and ripening fruits studied in situ with reflectance spectroscopy. Physiol Plant. 1998;104:661-7.

52. Shi J, Maguer ML. Lycopene in tomatoes: chemical and physical properties affected by food processing. Crit Rev Biotechnol. 2000;20:293-334.

53. Ramel F, Mialoundama AS, Havaux M. Nonenzymic carotenoid oxidation and photooxidative stress signalling in plants. J Exp Bot. 2013;64:799-805.

54. Mattila H, Khorobrykh S, Havurinne V, Tyystjärvi E. Reactive oxygen species: reactions and detection from photosynthetic tissues. J Photochem Photobiol B. 2015;152:176-214.

55. Havaux M. Carotenoid oxidation products as stress signals in plants. Plant J. 2014;79:597-606

56. Dong Y, Li B, Malitsky S, Rogachev I, Aharoni A, Kaftan F, Svatoš A, Franceschi P. Sample preparation for mass spectrometry imaging of plant tissues: a review. Front Plant Sci. 2016;7:60

57. Gruszecki WI, Zelent B, Leblanc RM. Fluorescence of zeaxanthin and violaxanthin in aggregated forms. Chem Phys Lett. 1990;171:563-8.

\footnotetext{
Ready to submit your research? Choose BMC and benefit from:

- fast, convenient online submission

- thorough peer review by experienced researchers in your field

- rapid publication on acceptance

- support for research data, including large and complex data types

- gold Open Access which fosters wider collaboration and increased citations

- maximum visibility for your research: over 100M website views per year
}

At BMC, research is always in progress.

Learn more biomedcentral.com/submissions 